\title{
DESARROLLO DE UN SISTEMA DE ANÁLISIS FISICOQUÍMICO BASADO EN ESPECTROSCOPÍA DE EMISIÓN ATÓMICA INDUCIDA POR RADIACIÓN LÁSER
}

\section{Oscar Urquidi y Omar Ormachea}

\section{RESUMEN}

Se presenta el desarrollo de un sistema de análisis fisicoquímico basado en espectroscopia de emisión atómica inducida por radiación láser, o LIBS por sus siglas en ingles. Se desarrolló el sistema LIBS compuesto de un láser Nd:YAG +++ portátil de una potencia estimada de $10 \mathrm{MW} / \mathrm{cm}^{2}$, un espectrómetro de difracción de $0.27 \mathrm{~nm}$ de resolución y $76.9 \mathrm{~nm}$ de ancho espectral, un sistema de entrega y recolección de luz, un sistema de coordinación y el programa de análisis espectral para el análisis de los datos obtenidos por el espectrómetro, todos estos elementos desarrollados en el CIO. El sistema de entrega y recolección de luz es el encargado de focalizar la radiación láser para producir el plasma y luego recoger la luz emitida y enviarla por una fibra óptica hacia el espectrómetro. Este último se desarrolló en base a una lente focalizadora de radiación láser, una lente colectora acoplada a una fibra óptica y los elementos optomecánicos para un posicionamiento preciso del área de medición. El programa de análisis espectral basado en Matlab permitió realizar comparaciones con espectros LIBS conocidos, buscar coincidencias y establecer la presencia de distintos elementos en los espectros analizados. Los resultados fueron satisfactorios, logrando la identificación de $\mathrm{Cu}$ y Li en distintas muestras analizadas. Finalmente, se elaboró una propuesta para una versión portátil del sistema desarrollado, demostrando su capacidad para realizar trabajos de campo en las áreas de minería y geoquímica.

Palabras Clave: Espectroscopia de Emisión Atómica, LIBS, Análisis Físico Químico, Análisis Espectral. 